\title{
THE MULTIPLICATION TABLE FOR SMOOTH INTEGERS
}

\author{
MARZIEH MEHDIZADEH
}

\begin{abstract}
The Erdös multiplication table problem asks what is the number of distinct integers appearing in the $N \times N$ multiplication table. The order of magnitude of this quantity was determined by Ford [7]. In this paper we study the number of $y$-smooth entries of the $N \times N$ multiplication table that is to say entries with no prime factors greater than $y$.
\end{abstract}

\section{INTRODUCTION}

The multiplication table problem involves estimating

$$
A(x):=\#\{a b: a, b \leq \sqrt{x}, \quad \text { and } \quad a, b \in \mathbb{N}\} .
$$

This interesting question, posed by Erdős, has been studied by many authors. Erdős in [6], showed that for all $\varepsilon>0$, we have

$$
\frac{x}{(\log x)^{\delta+\epsilon}} \leq A(x) \leq \frac{x}{(\log x)^{\delta-\varepsilon}} \quad(x \rightarrow \infty),
$$

where

$$
\delta=1-\frac{1+\log \log 2}{\log 2}=0.0860 \ldots .
$$

The best estimate of $A(x)$ is a result due to Kevin Ford [7]. He proved the following estimate, that significantly improved the order of magnitude of $A(x)$ as follows

$$
A(x) \asymp \frac{x}{(\log x)^{\delta}(\log \log x)^{3 / 2}} .
$$

Notation: In this paper, we use the notation $f(x) \asymp g(x)$ if both $f(x) \ll g(x)$ and $g(x) \ll f(x)$ hold, where $f(x) \ll g(x)$ or $f(x)=O(g(x))$ interchangeably to mean that $|f(x)| \leq c g(x)$ holds with some constant $c$ for all $x$ in a range which will normally be clear from the context. Also, the notation $f(x) \sim g(x)$ means that $f(x) / g(x) \rightarrow 1$ as $x \rightarrow \infty$, and $f(x)=o(g(x))$ means that $f(x) / g(x) \rightarrow 0$ as $x \rightarrow \infty$.

Also, $u$ is defined as

$$
u:=\frac{\log x}{\log y} \quad x \geq y \geq 2,
$$

and we let $\log _{k} x$ denote the $k$-fold iterated $\log$ arithm, defined by $\log _{1} x:=\log x$ and $\log _{k} x=$ $\log \log _{k-1} x$, for $k>1$. 
Motivated by this background, in this paper we investigate the multiplication table problem for smooth integers. The set of $y$-smooth numbers, is defined by

$$
S(x, y):=\{n \leq x: P(n) \leq y\},
$$

where $P(n)$ denotes the largest prime factor of an integer $n \geq 2$, with the convention $P(1)=1$. Set

$$
\Psi(x, y):=|S(x, y)| .
$$

Our main aim in this work is to study

$$
A(x, y):=\#\{a b: a, b \in S(\sqrt{x}, y)\} .
$$

Hence computing $A(x, y)$ is equivalent to estimating the size of $S(\sqrt{x}, y) \cdot S(\sqrt{x}, y)$.

A simple approximation of $\Psi(x, y)$ proved by Canfield, Erdös and Pomerance [3] states that for a fixed $\epsilon>0$, we have

$$
\Psi(x, y)=x u^{-u(1+o(1))} \quad \text { as } \quad u \rightarrow \infty,
$$

for $u \leq y^{1-\epsilon}$, that is $y \geq(\log x)^{1+\epsilon}$.

By estimate (4), one can see that for $u$ large (or $y$ small), the value of $\Psi(x, y)$ is small. It counts the integers having large number of prime factors. Since in this case every $n$ has a lot of small prime factors, we can find $a$ and $b$ such that $n=a b$ and $a, b \leq \sqrt{x}$.

If $u$ is small (which means that $y$ is large), then by (4), one can deduce that the value of $\Psi(x, y)$ is large compared to $x$. In this case, $S(x, y)$ contains integers with large prime factors and we expect the size of $S(\sqrt{x}, y) \cdot S(\sqrt{x}, y)$ to be small.

It is good to mention that by a connection to sum-product problem, Banks and Covert [2] by invoking combinatorial tools, have considered the behaviour of $A\left(x^{2}, y\right)=|S(x, y) \cdot S(x, y)|$ in different ranges of $y$, particularly for the cases when $y$ is relatively small or large.

Here we present a simple idea to prove that $A(x, y)$ has a same size as $\Psi(x, y)$ when $y$ is small compared to $\log x$. Let $n \leq \frac{x}{y}$ be a $y$-smooth number. If $n \leq \sqrt{x}$ then trivially we have $n \in A(x, y)$. Thus, we assume that $\sqrt{x} \leq n$. Let $p_{1} \leq p_{2} \leq \cdots \leq p_{k}$ be prime factors of $n$. Consider the following sequence obtained by prime factors of $n$ :

$$
n_{0}=1, \quad n_{j}=\prod_{i=1}^{j} p_{i}, \quad 1 \leq j \leq k .
$$

Since $n \geq \sqrt{x}$ then there exists a unique integer $s$, with $0 \leq s<k$ such that $n_{s}<\sqrt{x} \leq n_{s+1}$. Each prime factor of $n$ is less than $y$, therefore

$$
n_{s} \leq \sqrt{x} \leq n_{s+1} \leq n_{s} y
$$

Set $d=n_{s}$, then

$$
\frac{\sqrt{x}}{y} \leq d \leq \sqrt{x}
$$

Since $n \leq x / y$, then we easily conclude that

$$
\frac{n}{d} \leq \sqrt{x}
$$


Therefore,

$$
\Psi(x / y, y) \leq A(x, y) \leq \Psi(x, y)
$$

and by a simple argument one can deduce that as $x, y \rightarrow \infty$ then $\Psi(x / y, y) \sim \Psi(x, y)$ when $y=o(\log x)$, (see Lemma 2.4). This argument leads us to state the following theorem.

Theorem 1.1. If $y=o(\log x)$ then we have

$$
A(x, y) \sim \Psi(x, y) \quad \text { as } \quad x, y \rightarrow \infty .
$$

The problem gets harder, and hence, more interesting when $y$ takes larger values compared to $\log x$. We shall prove the following theorem for small values of $y$ compared to $x$.

Theorem 1.2. We have

$$
A(x, y) \sim \Psi(x, y) \quad \text { as } \quad x, y \rightarrow \infty,
$$

when $u$ and $y$ satisfy the range

$$
\frac{u \log u}{\left(\log y \log _{2} y \log _{3} y\right)^{2}} \rightarrow \infty, \quad \text { which implies, } y \leq \exp \left\{\frac{(\log x)^{1 / 3}}{\left(\log _{2} x\right)^{1 / 3+\epsilon}}\right\},
$$

for $\epsilon>0$ arbitrarily small.

Theorem 1.2 is proved in Section 3. The proof relies on some probabilistic arguments and recent estimates for $\Psi(x / p, y)$ where $p$ is a prime factor of $n$.

If $y$ takes values very close to $x$, which implies $u$ is small compared to $\log \log y$, then we will show the following theorem.

Theorem 1.3. Let $\epsilon>0$ is arbitrarily small, then we have

$$
A(x, y)=o(\Psi(x, y)) \quad \text { as } \quad x, y \rightarrow \infty,
$$

where $u$ and $y$ satisfying the range

$$
u<(L-\epsilon) \log _{2} y, \quad \text { which implies, } y \geq \exp \left\{\frac{\log x}{(L-\epsilon) \log _{2} x}\right\},
$$

where $L:=\frac{1-\log 2}{\log 2}$.

Theorem 1.3 is proved in Section 4, by applying an Erdős' idea [5], suitably modified for $y$-smooth integers.

In what follows, we will give a heuristic argument that predicts the behaviour of $A(x, y)$ in ranges (5) and (6).

We define the function $\tau(n ; A, B)$ to be the number of all divisors of $n$ in the interval $(A, B]$. In other words.

$$
\tau(n ; A, B):=\#\{d: d \mid n \Rightarrow A<d \leq B\} .
$$

Let $n \in S((1-\eta) x, y)$ be a square-free number with $k$ prime factors, where $\eta \rightarrow 0$ as $x \rightarrow \infty$. Assume that the set

$$
D(n):=\{\log d: d \mid n\}
$$


is uniformly distributed in the interval $[0, \log n]$. So

$$
P(d \in(A, B)):=\tau(n) \frac{\log B-\log A}{\log n},
$$

where the sample space is defined by

$$
S:=\{n \leq x: \omega(n)=k\},
$$

and $n$ being chosen uniformly at random. By this assumption, the expected value of the function $\tau(n,(1-\eta) \sqrt{x}, \sqrt{x})$ is as follows

$$
\mathbb{E}[\tau(n,(1-\eta) \sqrt{x}, \sqrt{x})]=\frac{2^{k} \log (1 /(1-\eta))}{\log \sqrt{x}} \asymp \frac{2^{k}}{u \log y} .
$$

Alladi and Hildebrand in [1] and [11] showed that the normal number of prime factors of $y$-smooth integers is very close to its expected value $u+\log _{2} y$ in different ranges of $y$. Hence, from (8), we deduce that

$$
\mathbb{E}[\tau(n,(1-\eta) \sqrt{x}, \sqrt{x})] \asymp \frac{2^{u+\log _{2} y}}{\log y} .
$$

If $2^{u+\log _{2} y} / \log y \rightarrow \infty$, then we expect that $n$ will have a divisor $d$ in the interval $((1-\eta) \sqrt{x}, \sqrt{x}$. We know $n \leq(1-\eta) x$. Thus, $n / d \leq \sqrt{x}$, and we can deduce that $n \in A(x, y)$, this means that

$$
\Psi((1-\eta) x, y) \leq A(x, y) .
$$

Trivially $A(x, y) \leq \Psi(x, y)$. So by this argument, we obtain

$$
A(x, y) \sim \Psi(x, y)
$$

when $\eta \rightarrow 0$ as $x \rightarrow \infty$.

On the other hand, if $2^{u+\log _{2} y} / \log y \rightarrow 0$, then we expect that none of integers in $S((1-\eta) x, y)$ have a divisor in $((1-\eta) \sqrt{x}, \sqrt{x}]$ (except a set with density 0$)$, this means that

$$
A(x, y)=o(\Psi(x, y)) \quad \text { as } \quad x, y \rightarrow \infty .
$$

This heuristic gives an evidence for the following conjecture:

Conjecture 1. If $L:=\frac{1-\log 2}{\log 2}$, then we have the following dichotomy

(1) : If $u-L \log _{2} y \rightarrow+\infty$, which implies

$$
y \leq \exp \left\{\frac{\log x}{L \log _{2} x}\right\},
$$

Then, we have

$$
A(x, y) \sim \Psi(x, y) \quad \text { as } \quad x, y \rightarrow \infty .
$$

(2) : If $u-L \log _{2} y \rightarrow-\infty$, which implies that for small $\epsilon>0$

$$
y \geq \exp \left\{\frac{\log x}{(L-\epsilon) \log _{2} x}\right\},
$$

Then, we have

$$
A(x, y)=o(\Psi(x, y)) \quad \text { as } \quad x, y \rightarrow \infty .
$$


Theorem 1.2 and Theorem 1.3 are in the direction of the first case and the second case of Conjecture (1) respectively, but the claimed ranges in the conjecture are stronger than the claimed ranges in Theorem 1.2 and Theorem 1.3, and the reason stems from uniformity assumption about $D(n)$.

\section{Acknowledgement}

I would like to thank Andrew Granville and Dimitris Koukoulopoulos for all their advice and encouragement as well as their valuable comments on the earlier version of the present paper. I am also grateful to Sary Drappeau, Farzad Aryan and Oleksiy Klurman for helpful conversations.

\section{Preliminaries}

In this section, we review some results used in the proof of our main theorems. We first fix some notation. In this chapter $\rho(u)$ is the Dickman-de Bruijn function, as we defined in the introduction. By $[9,3.9]$ we have the following estimate for $\rho(u)$

$$
\rho(u)=\left(\frac{e+o(1)}{u \log u}\right)^{u} \quad \text { as } \quad u \rightarrow \infty .
$$

Theorem 2.1 (Hildebrand [10]). The estimate

$$
\Psi(x, y)=x \rho(u)\left(1+O_{\epsilon}\left(\frac{\log (u+1)}{\log y}\right)\right)
$$

holds uniformly in the range

$$
x \geq 3, \quad 1 \leq u \leq \frac{\log x}{\left(\log _{2} x\right)^{\frac{5}{3}+\epsilon}}, \quad \text { that is, } y \geq \exp \left(\left(\log _{2} x\right)^{\frac{5}{3}+\epsilon}\right),
$$

where $\epsilon$ is any fixed positive number.

Combining (10) with the asymptotic formula (9), one can arrive at the following simple corollary

Corollary 2.2. We have

$$
\Psi(x, y)=x u^{-(u+o(u))},
$$

as $y$ and $u$ tend to infinity, uniformly in the range (11), for any fixed $\epsilon>0$.

We will apply this estimate in the proof of Theorem 1.3. However this estimate of $\Psi(x, y)$ is not very sharp for large values of $u$, for which the saddle point method is more effective.

Let $\alpha:=\alpha(x, y)$ be a real number satisfying

$$
\sum_{p \leq y} \frac{\log p}{p^{\alpha}-1}=\log x .
$$

One can show that $\alpha$ is unique. This function will play an essential role in this work, so we briefly recall some fundamental facts of this function that are used frequently. By [4, Lemma 3.1] we have the following estimates for $\alpha$. 


$$
\alpha(x, y)=\frac{\log (1+y / \log x)}{\log y}\left\{1+O\left(\frac{\log _{2} y}{\log y}\right)\right\} \quad x \geq y \geq 2 .
$$

For any $\epsilon>0$, we have the particular cases

$$
\alpha(x, y)=1-\frac{\xi(u)}{\log y}+O\left(\frac{1}{L_{\epsilon}(y)}+\frac{1}{u(\log y)^{2}}\right) \quad \text { if } y \geq(\log x)^{1+\epsilon},
$$

where

$$
L_{\epsilon}(y)=\exp \left\{(\log y)^{3 / 5-\epsilon}\right\},
$$

and $\xi(t)$ is the unique real non-zero root of the equation

$$
e^{\xi(t)}=1+t \xi(t)
$$

Also for small values of $y$, we have

$$
\alpha(x, y)=\frac{\log \left(1+\frac{y}{\log x}\right)}{\log y}\left\{1+O\left(\frac{1}{\log y}\right)\right\} \text { if } 2 \leq y \leq(\log x)^{2} .
$$

We now turn to another ingredient related to the behaviour of $\Psi(x, y)$. The following estimate is a special case of a general result of de La Breteche and Tenenbaum [4, Theorem 2.4].

Theorem 2.3. If $d \leq y$, then uniformly for $x \geq y \geq 2$ we have

$$
\Psi(x / d, y)=\left\{1+O\left(\frac{1}{u}+\frac{\log y}{y}\right)\right\} \frac{\Psi(x, y)}{d^{\alpha}} .
$$

We can deduce the following lemma by Theorem 2.3 which completes the proof of Theorem 1.1

Lemma 2.4. If $y \geq 2$ and $y=o(\log x)$, then we have

$$
\Psi(x / y, y) \sim \Psi(x, y) \text { as } x \rightarrow \infty .
$$

Proof. $(i)$ : Let $y \geq\left(\log _{2} x\right)^{2}$ and $y=o(\log x)$. By applying (18), if $d=y$, we obtain

$$
\Psi(x / y, y)=\frac{\Psi(x, y)}{y^{\alpha}}\left\{1+O\left(\frac{\log y}{y}\right)\right\} .
$$

By combination of the above estimate along with (17), we get

$$
\Psi(x / y, y)=\frac{\Psi(x, y)}{\left(1+\frac{y}{\log x}\right)^{1+O\left(\frac{1}{\log y}\right)}}\left\{1+O\left(\frac{\log y}{y}\right)\right\} .
$$

We remark again that $y=o(\log x)$, so we obtain

$$
\frac{1}{(1+y / \log x)^{1+O(1 / \log y)}} \rightarrow 1 \quad \text { when } \quad x \rightarrow \infty .
$$

Also, we have

$$
\frac{\log y}{y} \rightarrow 0 \text { when } \quad x \rightarrow \infty
$$


since $y \geq\left(\log _{2} x\right)^{2}$. Thus, by (21), we conclude

$$
\frac{\Psi(x / y, y)}{\Psi(x, y)} \rightarrow 1 \quad \text { when } \quad x \rightarrow \infty .
$$

(ii) : Let $2 \leq y \leq\left(\log _{2} x\right)^{2}$, then by recalling Ennola's theorem ??, we get

$$
\begin{aligned}
\Psi(x / y, y) & =\frac{1}{\pi(y) !} \prod_{p \leq y} \frac{\log x / y}{\log p}\left\{1+O\left(\frac{y^{2}}{\log x \log y}\right)\right\} \\
& =\frac{1}{\pi(y) !} \prod_{p \leq y} \frac{\log x}{\log p} \prod_{p \leq y}\left(1-\frac{\log y}{\log x}\right)\left\{1+O\left(\frac{y^{2}}{\log x \log y}\right)\right\} \\
& =\Psi(x, y)\left(1+O\left(\pi(y) \frac{\log y}{\log x}\right)\right) \\
& =\Psi(x, y)\left(1+O\left(\frac{y}{\log x}\right)\right)
\end{aligned}
$$

which gives that

$$
\Psi(x / y, y) \sim \Psi(x, y) \text { as } \quad x \rightarrow \infty,
$$

and this completes the proof.

Finally, we define

$$
\theta(x, y, z):=\#\{n \leq x: p \mid n \Rightarrow z \leq p \leq y\} .
$$

This function has been studied extensively in the literature. Namely Friedlander [8] and Saias $[13,14]$ gave several estimates for $\theta(x, y, z)$ in different ranges. The following theorem is due to Saias [14, Theorem 5] which is used in Section 4.

Theorem 2.5. There exists a constant $c>0$ such that for $x \geq y \geq z \geq 2$ we have

$$
\theta(x, y, z) \leq c \frac{\Psi(x, y)}{\log z}
$$

\section{Proof of TheOrem 1.2}

We begin this section by setting some notation. Let $\eta$ be defined by

$$
\eta:=\frac{1}{\log _{3} y}
$$

and set

$$
N:=\left\lfloor\frac{\log _{2} y-\log \eta}{\log 2}+2\right\rfloor,
$$

which play an essential role in process of the proof.

The idea of the proof of Theorem 1.2 is a combination of some probabilistic and combinatorial techniques. Before going through the details, we give a sketch of proof here. 
The first step of proving Theorem 1.2 is to study the number of all prime factors of $n$ in the narrow intervals

$$
J_{i}:=\left[(1-\kappa) y^{1-\frac{1}{2^{i}}}, y^{1-\frac{1}{2^{i}}}\right], \quad 1 \leq i \leq N,
$$

of multiplicative length $(1-\kappa)^{-1}$, where $\kappa$ is defined as

$$
\kappa:=\frac{\eta}{2 N} \text {. }
$$

Also, we define the tail interval

$$
J_{\infty}:=[(1-\kappa) y, y]
$$

Let $\omega_{i}(n)$ be the number of prime factors of $n$ in $J_{i}$ for each $i \in\{1,2, \ldots, N, \infty\}$, more formally

$$
\omega_{i}(n):=\#\left\{p \mid n: p \in J_{i}\right\} .
$$

We define $\mu_{i}(x, y)$ to be the expectation of $\omega_{i}(n)$, defined by

$$
\mu_{i}(x, y):=\frac{1}{\Psi(x, y)} \sum_{n \in S(x, y)} \omega_{i}(n)
$$

In Proposition 3.4, we will prove that for almost all $y$-smooth integers the value of $\omega_{i}(n)$ exceeds $\mu_{i}(x, y) / 2$. We establish this by applying the Chebyshev's inequality

$$
\frac{\#\left\{n \in S(x, y): \omega_{i}(n) \leq \mu_{i}(x, y) / 2\right\}}{\Psi(x, y)} \leq \frac{4 \sigma_{i}^{2}(x, y)}{\mu_{i}^{2}(x, y)},
$$

where

$$
\sigma_{i}^{2}(x, y):=\frac{1}{\Psi(x, y)} \sum_{n \in S(x, y)}\left(\omega_{i}(n)-\mu_{i}(x, y)\right)^{2},
$$

is the variance of $\omega_{i}(n)$ and $i \in\{1,2, \ldots, N, \infty\}$. We will conclude that there is at least one prime factor $p_{i}$ in each $J_{i}$ for $1 \leq i \leq N$ and $N$ prime factors $q_{1}, \ldots, q_{N}$ in $J_{\infty}$. Then by using the product of these prime factors in Corollary 3.5, we will find a divisor $D_{j}$ of $n$ such that

$$
(1-\kappa)^{N} y^{N-j / 2^{N}} \leq D_{j} \leq y^{N-j / 2^{N}},
$$

for an integer $j$ in $\left\{0,1, \ldots 2^{N}-1\right\}$.

Then, we fix an integer $n$ in $S((1-\eta) x, y)$, and by defining $m:=\frac{n}{\prod_{i=1}^{N} p_{i} q_{i}}$, we will easily show that there is a divisor $d_{j}$ of $n$, such that

$$
\frac{\sqrt{n}}{y^{N}} y^{j / 2^{N}}<d_{j}<\frac{\sqrt{n}}{y^{N}} y^{(j+1) / 2^{N}} .
$$

Multiplying $D_{j}$ and $d_{j}$ and using the definitions of $\eta, \kappa$ and $N$, gives a new divisor $d$ of $n$ that helps us to write $n$ as the product of two divisors less than $\sqrt{x}$.

Before stating technical lemmas we get an estimate for the expected value of $\omega_{i}(n)$ for all $1 \leq i \leq N$ and $i=\infty$. By changing the order of summation in (27), we can easily see that

$$
\mu_{i}(x, y)=\sum_{p \in J_{i}} \frac{\Psi(x / p, y)}{\Psi(x, y)} .
$$


By (18), we have the following estimate

$$
\mu_{i}(x, y)=\sum_{p \in J_{i}} \frac{1}{p^{\alpha}}\left(1+O\left(\frac{1}{u}+\frac{\log y}{y}\right)\right),
$$

for all $1 \leq i \leq N$ and $x \geq y \geq 2$. Also, we obtain the following estimate for $\mu_{i}(x / q, y)$, where $q$ is a prime divisor of $n$.

$$
\mu_{i}(x / q, y)=\sum_{p \in J_{i}} \frac{1}{p^{\alpha_{q}}}\left\{1+O\left(\frac{1}{u_{q}}+\frac{\log y}{y}\right)\right\},
$$

where $u_{q}:=u-\log q / \log y$. By substitution we obtain $x / q=y^{u_{q}}$. Set the saddle point $\alpha_{q}:=\alpha(x / q, y)$, defined as the unique real number satisfying in

$$
\sum_{p \leq y} \frac{\log p}{p^{\alpha_{q}}-1}=\log (x / q) .
$$

We are ready to prove the following lemma that shows the difference between $\mu_{i}(x / q, y)$ and $\mu_{i}(x, y)$ is small.

Lemma 3.1. Let $q$ be a prime divisor of $n \in S(x, y)$, then we have

$$
\left|\mu_{i}(x / q, y)-\mu_{i}(x, y)\right| \ll \frac{\mu_{i}(x, y)}{u} .
$$

Proof. We use the estimate

$$
0<-\alpha^{\prime}(u):=-\frac{d \alpha(u)}{d u} \asymp \frac{\bar{u}}{u^{2} \log y},
$$

established in [12, formula 6.6], where $\bar{u}:=\min \left\{u, \frac{y}{\log y}\right\}$. By (34), we deduce

$$
\left|\alpha^{\prime}(u)\right| \ll \frac{1}{u \log y}
$$

Then applying (35), gives that

$$
\begin{aligned}
\alpha-\alpha_{q} \leq \int_{u_{q}}^{u}\left|\alpha^{\prime}(v)\right| d v & \ll \int_{u}^{u_{q}} \frac{d v}{v \log y} \\
& =\frac{1}{\log y} \log \left(\frac{u}{u_{q}}\right) \asymp \frac{\log q}{\log y \log x} .
\end{aligned}
$$

By expanding $\mu_{i}(x / q, y)-\mu_{i}(x, y)$ and using (30) and (32), we get

$$
\begin{aligned}
\left|\mu_{i}(x / q, y)-\mu_{i}(x, y)\right| & =\left|\sum_{p \in J_{i}}\left(\frac{\Psi(x / p q, y)}{\Psi(x / q, y)}-\frac{\Psi(x / p, y)}{\Psi(x, y)}\right)\right| \\
& \leq \sum_{p \in J_{i}} \frac{1}{p^{\alpha}}\left\{\left|p^{\alpha-\alpha_{q}}-1\right|+O\left(\frac{1}{u}+\frac{\log y}{y}\right)\right\} .
\end{aligned}
$$


By the Taylor expansion of the exponential function and invoking (36) we obtain

$$
\exp \left\{\left(\alpha-\alpha_{q}\right) \log p\right\}-1 \ll \frac{\log p \log q}{\log y \log x} .
$$

We recall that $p, q \leq y$ for $1 \leq i \leq N$ and $i=\infty$. From this we infer that

$$
\left|p^{\alpha-\alpha_{q}}-1\right| \ll \frac{1}{u}
$$

this finishes the proof.

In the following lemma we shall find an upper bound for $\sigma_{i}^{2}(x, y)$ (defined in (29)) for each $i \in\{1,2, \ldots, N, \infty\}$.

Lemma 3.2. We have

$$
\sigma_{i}^{2}(x, y) \ll \mu_{i}(x, y)+\mu_{i}^{2}(x, y) / u,
$$

where $i \in\{1,2, \ldots, N, \infty\}$.

Proof. By the definition of $\sigma_{i}^{2}(x, y)$ in (29), we have

$$
\sigma_{i}^{2}(x, y)=\frac{1}{\Psi(x, y)} \sum_{n \in S(x, y)}\left[\omega_{i}^{2}(n)-2 \mu_{i}(x, y) \omega_{i}(n)+\mu_{i}^{2}(x, y)\right]
$$

Using the definition of $\omega_{i}(n)$ in $(26)$, gives

$$
\sum_{n \in S(x, y)} \omega_{i}(n)=\sum_{n \in S(x, y)} \sum_{p \in J_{i}} \mathbb{1}_{p \mid n}=\sum_{p \in J_{i}} \Psi(x / p, y),
$$

where the indicator function $\mathbb{1}_{p \mid n}$ is 1 or 0 according to the prime $p$ divides $n$ or not. By the definition of $\mu_{i}(x, y)$ in $(30)$, one can deduce that

$$
\sum_{n \in S(x, y)} \omega_{i}(n)=\Psi(x, y) \mu_{i}(x, y)
$$

By applying (30) and the equation above, we obtain

$$
\begin{aligned}
\Psi(x, y) \sigma_{i}^{2}(x, y) & =\sum_{n \in S(x, y)}\left[\omega_{i}^{2}(n)-2 \mu_{i}(x, y) \omega_{i}(n)+\mu_{i}^{2}(x, y)\right] \\
& =\sum_{n \in S(x, y)} \omega_{i}^{2}(n)-2 \Psi(x, y) \mu_{i}^{2}(x, y)+\psi(x, y) \mu_{i}^{2}(x, y) \\
& =\left(\sum_{\substack{p, q \in J_{j} \\
p \neq q}} \Psi(x / p q, y)\right)-\Psi(x, y) \mu_{i}^{2}(x, y)+\sum_{p \in J_{i}} \Psi(x / p, y) \\
& :=S_{1}+S_{2},
\end{aligned}
$$


where $S_{1}:=\sum_{\substack{p, q \in J_{j} \\ p \neq q}} \Psi(x / p q, y)-\Psi(x, y) \mu_{i}^{2}(x, y)$ and $S_{2}:=\sum_{p \in J_{i}} \Psi(x / p, y)$. We next find an upper bound for each $S_{i}$. We first consider $S_{1}$, by using (30) we can get

$$
\sum_{\substack{p, q \in J_{i} \\ p \neq q}} \Psi(x / p q, y)-\Psi(x, y) \mu_{i}^{2}(x, y) \leq \sum_{p \in J_{i}} \Psi(x / p, y)\left(\mu_{i}(x / p, y)-\mu_{i}(x, y)\right) .
$$

By Lemma 3.1 and using (39), we obtain the following upper bound for $S_{1}$

$$
S_{1} \leq C \frac{\Psi(x, y) \mu_{i}^{2}(x, y)}{u}
$$

where $C$ is a positive constant. It remains to estimate $S_{2}$, from (30) we have

$$
S_{2}=\Psi(x, y) \mu_{i}(x, y) .
$$

By substituting the upper bounds for $S_{1}$ and $S_{2}$, we get

$$
\sigma_{i}^{2}(x, y)=\frac{S_{1}+S_{2}}{\Psi(x, y)} \ll\left(\mu_{i}(x, y)+\frac{\mu_{i}^{2}(x, y)}{u}\right),
$$

and the proof is complete.

Now we give an order of magnitude for $\mu_{i}(x, y)$, where $i \in\{1,2, \ldots, N, \infty\}$

Lemma 3.3. We have

$$
\mu_{i}(x, y) \asymp \kappa \frac{Y^{1-\frac{1}{2^{i}}}}{\log y}
$$

where $i \in\{1,2, \ldots, N, \infty\}$, and

$$
Y:=y^{1-\alpha}
$$

Proof. By the definition of each $J_{i}$, we obtain the following simple inequalities

$$
\frac{1}{y^{\alpha\left(1-1 / 2^{i}\right)}} \#\left\{p \in J_{i}\right\} \leq \sum_{p \in J_{i}} \frac{1}{p^{\alpha}} \leq \frac{1}{(1-\kappa) y^{\alpha\left(1-1 / 2^{i}\right)}} \#\left\{p \in J_{i}\right\} .
$$

By applying the prime number theorem, we obtain

$$
\begin{aligned}
\#\left\{p: p \in J_{i}\right\} & =\pi\left(y^{1-1 / 2^{i}}\right)-\pi\left((1-\kappa) y^{1-1 / 2^{i}}\right) \\
& =\frac{y^{1-1 / 2^{i}}}{\log \left(y^{1-1 / 2^{i}}\right)}-\frac{(1-\kappa) y^{1-1 / 2^{i}}}{\log \left((1-\kappa) y^{1-1 / 2^{i}}\right)}+O\left(\frac{y^{1-1 / 2^{i}}}{\log ^{2} y}\right) \\
& =\frac{y^{1-1 / 2^{i}}}{\left(1-1 / 2^{i}\right) \log y}-\frac{(1-\kappa) y^{1-1 / 2^{i}}}{\left(1-1 / 2^{i}\right) \log y}\left(1+O\left(\frac{\log (1-\kappa)}{\log y}\right)\right) \\
& =\frac{\kappa y^{1-1 / 2^{i}}}{\left(1-1 / 2^{i}\right) \log y}(1+o(1)),
\end{aligned}
$$

The last equality is true, since the given values of $\kappa$ and $N$ in (25) and (24) imply

$$
\kappa \asymp 1 /\left(\log _{2} y \log _{3} y\right) .
$$


By substituting (42) in (41) we have

$$
\mu_{i}(x, y) \asymp \kappa \frac{Y^{1-1 / 2^{i}}}{\log y},
$$

By the above lemmas, we are now ready for proving the following proposition.

Proposition 3.4. If $u$ and $y$ satisfy in range given in(5), we have

$$
\#\left\{n \in S(x, y): \omega_{i}(n)>\frac{\mu_{i}(x, y)}{2} \quad \forall i \in\{1, \ldots, N, \infty\}\right\} \sim \Psi(x, y) \quad \text { as } \quad x, y \rightarrow \infty \text {, }
$$

Proof. By the Chebyshev's inequality in (28) and using the upper bound for $\sigma_{i}^{2}(x, y)$ in lemma (3.2), we get

$$
\#\left\{n \in S(x, y): \omega_{i}(n) \leq \frac{\mu_{i}(x, y)}{2}\right\} \ll \Psi(x, y)\left(\frac{1}{\mu_{i}(x, y)}+\frac{1}{u}\right) .
$$

By the above inequality, we obtain an upper bound for the following set

$$
\begin{aligned}
M & :=\#\left\{n \in S(x, y): \exists i \in\{1, \ldots, N, \infty\} \quad \text { such that } \omega_{i}(n) \leq \frac{\mu_{i}(x, y)}{2}\right\} \\
& \ll \Psi(x, y)\left[\frac{1}{\mu_{\infty}(x, y)}+\frac{N}{u}+\sum_{i=1}^{N} \frac{1}{\mu_{i}(x, y)}\right] .
\end{aligned}
$$

Our main task that finishes the proof is to find a range such that $M / \Psi(x, y)$ tends to 0 . By using Lemma 3.3 and substituting the order of magnitude of $\mu_{i}(x, y)$ in (45), we get

$$
M \ll \Psi(x, y)\left[\frac{\log y}{\kappa Y}+\frac{N}{u}+\frac{\log y}{\kappa} \sum_{i=1}^{N} \frac{1}{Y^{1-1 / 2^{i}}}\right] .
$$

In what follows, we find a lower bound for $Y$ in two different ranges of $y$

(i) : If $y \leq(\log x)^{2}$, then by (17) $\alpha \leq 1 / 2+o(1)$ as $y \rightarrow \infty$. Therefore,

$$
Y \geq y^{1 / 2-o(1)} \geq y^{1 / 3} .
$$

By substituting this lower bound in (46) and using the precise value of $N$ in (24), we have

$$
\begin{aligned}
M & \ll \Psi(x, y)\left[\frac{\log y}{\kappa y^{1 / 3}}+\frac{N}{u}+\frac{\log y}{\kappa y^{1 / 3}} \sum_{i=1}^{N} y^{1 / 3\left(2^{i}\right)}\right] \\
& \ll \Psi(x, y)\left[\frac{\log _{2} y}{u}+\frac{y^{1 / 6} \log y}{\kappa y^{1 / 3}}\left(1+O\left(N y^{-1 / 12}\right)\right)\right] \\
& \ll \Psi(x, y) \frac{\log y}{\kappa y^{1 / 6}}
\end{aligned}
$$


By using the asymptotic value of $\kappa$ in (43), we obtain

$$
M \ll \Psi(x, y) \frac{\log y \log _{2} y \log _{3} y}{y^{1 / 6}},
$$

and clearly we have

$$
M=o(\Psi(x, y)) \quad \text { as } \quad x, y \rightarrow \infty,
$$

this finishes the proof for the case $y \leq(\log x)^{2}$.

(ii) : If $y \geq(\log x)^{2}$, by applying (14), we have

$$
1-\alpha=\frac{\xi(u)}{\log y}+O\left(\frac{1}{L_{\epsilon}(y)}+\frac{1}{u(\log y)^{2}}\right) .
$$

Using [15, Lemma 8.1], we have the following estimate of $\xi$

$$
\xi(t)=\log (t \log t)+O\left(\frac{\log _{2} t}{\log t}\right) \quad \text { if } \quad t>3
$$

Therefore,

$$
1-\alpha=\frac{\log (u \log u)}{\log y}+O\left(\frac{\log _{2} u}{\log y \log u}\right)
$$

Thus, we get

$$
\begin{aligned}
Y & =u \log u\left[1+O\left(\frac{\log _{2} u}{\log u}\right)\right] \\
& \asymp u \log u .
\end{aligned}
$$

By combining the above with the estimate in (49), and using the value of $N$ in (24), we get

$$
\begin{aligned}
M & \ll \Psi(x, y)\left[\frac{\log y}{\kappa u \log u}+\frac{N}{u}+\frac{\log y}{\kappa u \log u} \sum_{i=1}^{N}(u \log u)^{1 / 2^{i}}\right] \\
& \ll \Psi(x, y)\left[\frac{N}{u}+\frac{\log y}{\kappa u \log u}\left((u \log u)^{1 / 2}+(u \log u)^{1 / 2^{2}}+\ldots+(u \log u)^{1 / 2^{N}}\right)\right] \\
& \ll \Psi(x, y)\left[\frac{N}{u}+\frac{\log y}{\kappa(u \log u)^{1 / 2}}\left(1+O\left(N(u \log u)^{-1 / 4}\right)\right)\right] \\
& \ll \Psi(x, y)\left[\frac{\log _{2} y}{u}+\frac{\log y}{\kappa(u \log u)^{1 / 2}}\right],
\end{aligned}
$$

By using the order of $\kappa$ in (43), one can arrive at the following upper bound of $M$

$$
M \ll \Psi(x, y) \frac{\log y \log _{2} y \log _{3} y}{(u \log u)^{1 / 2}} .
$$

So there exists a constant $c$ such that for all $i \in\{1, \ldots, N, \infty\}$, we have

$$
\#\left\{n \in S(x, y): \omega_{i}(n)>\mu_{i}(x, y) / 2 \quad \forall i\right\} \geq \Psi(x, y)\left(1-c \frac{\log y \log _{2} y \log _{3} y}{(u \log u)^{1 / 2}}\right)
$$


and this finishes the proof by letting

$$
\frac{u \log u}{\left(\log y \log _{2} y \log _{3} y\right)^{2}} \rightarrow \infty .
$$

Corollary 3.5. If $x$ and $y$ satisfy the range (5), then almost all $n$ in $S(x, y)$ are divisible by at least one prime factor $p_{i}$ in $J_{i}$, and $N$ prime factors $q_{1}, \ldots, q_{N}$ in $J_{\infty}$. Moreover, the product $\prod_{i=1}^{N} p_{i} q_{i}$ has a divisor $D_{j}$ in each of intervals $\left[(1-\kappa)^{N} y^{N-j / 2^{N}}, y^{N-j / 2^{N}}\right]$, where $j \in$ $\left\{0,1, \ldots, 2^{N}-1\right\}$.

Proof. The first part of Corollary is a direct conclusion of Proposition 3.4.

For the second part, let $n$ be a $y$-smooth integer satisfying the first part of Corollary. We fix the following divisor of $n$

$$
D:=\prod_{i=1}^{N} p_{i} q_{i}
$$

where $p_{i} \in J_{i}$ and $q_{1}, \ldots, q_{N} \in J_{\infty}$.

Let $j$ be an arbitrary integer in $\left\{0,1, \ldots, 2^{N}-1\right\}$. Moreover, we define

$$
a_{0}:=N-\sum_{i=1}^{N} a_{i},
$$

where $a_{i}$ 's get the values 0 or 1 such that

$$
\sum_{i=1}^{N} \frac{a_{i}}{2^{i}}=j / 2^{N} .
$$

We now define the divisor of $D_{j}$ of $D$ with the following form

$$
D_{j}:=\prod_{i=1}^{N} p_{i}^{a_{i}} \prod_{i=1}^{a_{0}} q_{i},
$$

By using the bounds of $p_{i}$ s and $q_{i}$ s, one can get the following bounds for $D_{j}$.

$$
(1-\kappa)^{N} y^{N-\sum_{i=1}^{N} a_{i} / 2^{i}} \leq D_{j} \leq y^{N-\sum_{i=1}^{N} a_{i} / 2^{i}}
$$

By using (53), we have

$$
(1-\kappa)^{N} y^{N-j / 2^{N}} \leq D_{j} \leq y^{N-j / 2^{N}}
$$

and this finishes our proof.

We are ready now to prove Theorem 1.2.

Proof of Theorem 1.2. Let $n \leq(1-\eta) x$ be a $y$-smooth integer with at least one prime factor $p_{i}$ in each $J_{i}$, where $i=1, . ., N$, and $N$ prime divisors $q_{1}, q_{2}, \ldots, q_{N}$ in $J_{\infty}$. Set

$$
m:=\frac{n}{\prod_{i=1}^{N} p_{i} q_{i}} .
$$


By this definition, we get

when $4 N \leq u$. Thus,

$$
\frac{n}{\prod_{i=1}^{N} p_{i} q_{i}} \geq \frac{n}{y^{2 N}}>\sqrt{n}
$$

$$
m>\sqrt{n}
$$

Let $\left\{r_{v}\right\}$ be the increasing sequence of prime factors of $m$ and set $d_{v}=r_{1} \ldots r_{v}$.

Clearly, $m$ has at least one divisor bigger than $\frac{\sqrt{n}}{y^{N}}$. We suppose that $l$ is the smallest integer such that $d_{l} \geq \frac{\sqrt{n}}{y^{N}}$, and evidently we have

$$
d_{l-1} \leq \frac{\sqrt{n}}{y^{N}}
$$

So, we arrive at the following bounds for $d_{l}$

$$
\frac{\sqrt{n}}{y^{N}} \leq d_{l} \leq y d_{l-1} \leq \frac{\sqrt{n}}{y^{N-1}}
$$

We pick $k \in\left\{0,1,2 \ldots, 2^{N}-1\right\}$ such that

$$
\frac{\sqrt{n}}{y^{N}} y^{k / 2^{N}} \leq d_{l} \leq \frac{\sqrt{n}}{y^{N}} y^{(k+1) / 2^{N}} .
$$

By the second part of Corollary 3.5, for every $k$ in $\left\{0,1, \ldots, 2^{N}-1\right\}$ there exists a divisor $D_{k}$ such that

$$
(1-\kappa)^{N} y^{N-k / 2^{N}} \leq D_{k} \leq y^{N-k / 2^{N}},
$$

We define $d:=d_{l} D_{k}$, we have

$$
(1-\kappa)^{N} \sqrt{n} \leq d \leq y^{1 / 2^{N}} \sqrt{n}
$$

By using the values of $N$ in (24) and $\kappa$ in (25), we have

$$
e^{-\eta / 2} \sqrt{n} \leq d \leq e^{\eta / 2} \sqrt{n}
$$

Applying the Taylor expansion for exponential functions, gives

$$
\left(1-\eta+\frac{\eta^{2}}{2}+O\left(\eta^{3}\right)\right)^{1 / 2} \sqrt{n} \leq d \leq\left(1+\eta+\frac{\eta^{2}}{2}+O\left(\eta^{3}\right)\right)^{1 / 2} \sqrt{n}
$$

By using the assumption $n \leq(1-\eta) x$ in the upper bound and lower bound above, we obtain

$$
d \leq\left(1-\frac{\eta^{2}}{2}+O\left(\eta^{3}\right)\right)^{1 / 2} \sqrt{x} \leq \sqrt{x}
$$

and

$$
\frac{n}{d} \leq\left(1+\eta+\frac{\eta^{2}}{2}+O\left(\eta^{3}\right)\right)^{1 / 2} \sqrt{n} \leq\left(1-\frac{\eta^{2}}{2}+O\left(\eta^{3}\right)\right)^{1 / 2} \sqrt{x} \leq \sqrt{x} .
$$

Thus, we can write $n \in S((1-\eta) x, y)$ as the product of two divisors less than $\sqrt{x}$, and we can deduce that

By using (18), we have

$$
\Psi((1-\eta) x, y) \leq A(x, y) \leq \Psi(x, y)
$$




$$
\frac{\Psi((1-\eta) x, y)}{\Psi(x, y)}=(1-\eta)^{\alpha}\left\{1+O\left(\frac{1}{u}+\frac{\log y}{y}\right)\right\} \rightarrow 1 \quad \text { as } \quad x, y \rightarrow \infty
$$

this finishes the proof.

\section{Proof of Theorem1.3}

In this section, we shall study the behaviour of $A(x, y)$ for large values of $y$. When $y$ takes values very close to $x$, then the set of $y$-smooth integers contains integers having large prime factors. As we explained in the heuristic argument, one can expect that $A(x, y)=o(\Psi(x, y))$. To show this assertion, we recall the idea of Erdös used to prove the multiplication table problem for integers up to $x$.

We start our argument by giving an upper bound for $A^{*}(x)$, defined by

$$
A^{*}(x):=\#\{a b: a, b \leq \sqrt{x} \text { and }(a, b)=1\}
$$

We shall find an upper bound of $A^{*}(x)$ by considering the number of prime factors of $a$ and $b$. We first define

$$
\pi_{k}(x):=\#\{n \leq x: \omega(n)=k\}
$$

Therefore,

$$
\begin{aligned}
A^{*}(x) & \leq \sum_{k} \min \left\{\pi_{k}(x), \sum_{j=1}^{k-1} \pi_{j}(\sqrt{x}) \pi_{k-j}(\sqrt{x})\right\} \\
& \leq \sum_{k} \min \left\{\frac{c x}{\log x} \frac{\left(\log _{2} x\right)^{k-1}}{(k-1) !}, \sum_{j=1}^{k-1} \frac{c \sqrt{x}}{\log \sqrt{x}} \frac{\left(\log _{2} \sqrt{x}\right)^{j-1}}{(j-1) !} \frac{c \sqrt{x}}{\log \sqrt{x}} \frac{\left(\log _{2} \sqrt{x}\right)^{k-j-1}}{(k-j-1) !}\right\},
\end{aligned}
$$

where in the last inequality, we used the well-known result of Hardy and Ramanujan that states there are absolute constants $C$ and $c$ such that

$$
\pi_{k}(x) \leq \frac{c x}{\log x} \frac{\left(\log _{2} x+C\right)^{k-1}}{(k-1) !} \quad \text { for } \quad k=0,1,2, . . \quad \text { and } \quad x \geq 2 .
$$

By simplifying the upper bound in (58) and using Stirling's formula

$$
n ! \sim n^{n+\frac{1}{2}} e^{-n}
$$


we obtain

$$
\begin{aligned}
A^{*}(x) & \leq \sum_{k} \min \left\{\frac{c x}{\log x} \frac{\left(\log _{2} x\right)^{k-1}}{(k-1) !}, \frac{4 c^{2} x}{(\log x)^{2}} \sum_{j=0}^{k-2} \frac{1}{(k-2) !}\left(\begin{array}{c}
k-2 \\
j
\end{array}\right)\left(\log _{2} \sqrt{x}\right)^{k-2}\right\} \\
& =\sum_{k} \min \left\{\frac{c x}{\log x} \frac{\left(\log _{2} x\right)^{k-1}}{(k-1) !}, \frac{4 c^{2} x}{(\log x)^{2}} \frac{\left(2 \log _{2} \sqrt{x}\right)^{k-2}}{(k-2) !}\right\} \\
& =\sum_{k \leq \frac{\log _{2} x}{\log 2}} \frac{4 c^{2} x}{(\log x)^{2}} \frac{\left(2 \log _{2} \sqrt{x}\right)^{k-2}}{(k-2) !}+\sum_{k>\frac{\log _{2} x}{\log 2}} \frac{c x}{\log x} \frac{\left(\log _{2} x\right)^{k-1}}{(k-1) !} \\
& \ll \frac{x}{(\log x)^{1-\frac{1+\log \log 2}{\log 2}}\left(\log _{2} x\right)^{1 / 2}} \rightarrow 0 \quad \text { as } \quad x \rightarrow \infty .
\end{aligned}
$$

We shall get the same upper bound for $A(x)$. Let $n \leq x$ and there are $a$ and $b$ less than $\sqrt{x}$ such that $n=a b$. If $(a, b)=1$ then $n$ is counted by $A(x)$, and if $(a, b)=d>1$ then we can write $n$ as $n=a^{\prime} b^{\prime} d^{2}$ such that $\left(a^{\prime}, b^{\prime}\right)=1$. So, $\frac{n}{d^{2}} \leq \frac{x}{d^{2}}$, and $\frac{n}{d^{2}}$ will be counted by $A\left(\frac{x}{d^{2}}\right)$. Therefore,

$$
A(x) \leq \sum_{d \leq \sqrt{x}} A^{*}\left(\frac{x}{d^{2}}\right) \ll A^{*}(x)
$$

By (60), we get

Thus,

$$
A(x) \ll \frac{x}{(\log x)^{1-\frac{1+\log \log 2}{\log 2}}\left(\log _{2} x\right)^{1 / 2}} .
$$

$$
A(x)=o(x) \quad \text { as } \quad x \rightarrow \infty .
$$

Motivated by Erdős' idea for the multiplication table of integers up to $x$, we apply a similar method to find an upper bound for $A(x, y)$.

The first step of proof is to study the following function which plays a crucial role in this section. Let

$$
N_{k}(x, y, z):=\#\left\{n \in S(x, y): \Omega_{z}(n)=k\right\},
$$

where $\Omega_{z}(n)$ is the truncated version of $\Omega(n)$, only counting divisibility by primes not exceeding $z$ with their multiplicities. In other words

$$
\Omega_{z}(n):=\sum_{\substack{p^{v} \| n \\ p \leq z}} v
$$

In the following lemma, by using induction on $k$, we shall find an upper bound of type (59) for $N_{k}(x, y, z)$. The reason of applying truncation is to sieve out prime factors exceeding some power of $y$ which are the cause of big error terms as $k$ increases in each step of induction. The upper bound of $N_{k}(x, y, z)$ leads us to generalize Erdős' idea for $y$-smooth integers in a certain range of $y$. 
Lemma 4.1. Let $u \leq(C-\epsilon) \log \log y$, where $C$ is a positive constant and $\epsilon>0$ is arbitrarily small. Set the parameter $z$ such that

$$
\log \log z \ll u \text {. }
$$

Then, there are constants $A$ and $B$ such that the inequality

$$
N_{k}(x, y, z) \leq \frac{A \Psi(x, y)}{\log z} \frac{(\log \log z+B)^{k}}{k !}
$$

holds for every integer $k>0$.

Proof. When $k=0$, by (23), evidently we have

$$
N_{0}(x, y, z)=\theta(x, y, z) \leq c \frac{\Psi(x, y)}{\log z},
$$

where $c>0$ is a constant. When $k=1$, we can represent $n$ as $n=p m$, where $p \leq z$ and every prime factor $q$ of $m$ is between $z$ and $y$, then using the definition of $\theta(x, y, z)$ we have

$$
N_{1}(x, y, z)=\sum_{p \leq z} \sum_{\substack{m \leq x / p \\ q \mid m \Rightarrow z \leq q \leq y}} 1=\sum_{p \leq z} \theta(x / p, y, z) .
$$

By applying the estimate (18) and (23), there is constant $c$ such that

$$
N_{1}(x, y, z) \leq \sum_{p \leq z} \frac{c \Psi(x / p, y)}{\log z}=c \frac{\Psi(x, y)}{\log z} \sum_{p \leq z} \frac{1}{p^{\alpha}}\left\{1+O\left(\frac{1}{u}\right)\right\} .
$$

For the last summand we have

$$
\begin{aligned}
\sum_{p \leq z} \frac{1}{p^{\alpha}} & =\sum_{p \leq z} \frac{1}{p}\left(p^{1-\alpha}\right) \\
& =\sum_{p \leq z} \frac{1}{p}\{1+O((1-\alpha) \log p)\},
\end{aligned}
$$

since $(1-\alpha) \log p \leq(1-\alpha) \log z$, and $(1-\alpha) \log z$ is bounded in our range (see (64)). Therefore,

$$
\sum_{p \leq z} \frac{1}{p^{\alpha}}=\log _{2} z+O((1-\alpha) \log z)
$$

By using the estimate of $\alpha$ in (14) and the upper bound of $z$, we get

$$
(1-\alpha) \log z \ll \frac{\log u}{\log y} \log z \ll \frac{\log u}{\log _{2} y} \ll \frac{\log _{3} y}{\log _{2} y},
$$

and we obtain

$$
\sum_{p \leq z} \frac{1}{p^{\alpha}}=\log _{2} z+O\left(\frac{\log _{3} y}{\log _{2} y}\right)
$$


Thus,

$$
\sum_{p \leq z} \frac{1}{p^{\alpha}}\left\{1+O\left(\frac{1}{u}\right)\right\}=\log \log z+O(1),
$$

since we have $\log \log z \ll u$.

Substituting (66) in the upper bound of $N_{1}(x, y, z)$, gives

$$
N_{1}(x, y, z) \leq \frac{c \Psi(x, y)}{\log z}\left(\log _{2} z+O(1)\right) .
$$

We will show the lemma with $A=c$ and $B=O(1)$. We argue by induction: we assume that the estimate in (61) is true for any positive integer $k$, we now prove it for $n \in S(x, y)$ with $\Omega_{z}(n)=k+1$. There are $k+1$ ways to write $n$ as $n=p m_{1} m_{2}$ such that $p \leq z$ and $\Omega_{z}\left(m_{1}\right)=k$ and every prime factor of $m_{2}$ is greater than $z$. Then we have

$$
\begin{aligned}
N_{k+1}(x, y, z) & =\frac{1}{(k+1)} \sum_{p \leq z} \sum_{\substack{m_{1} \in S(x /(p), y) \\
\Omega_{z}\left(m_{1}\right)=k \\
m_{2} \in S\left(x /\left(p m_{1}\right), y\right) \\
q \mid m_{2} \Rightarrow q>z}} 1 \leq \frac{1}{(k+1)} \sum_{p \leq z} \sum_{m_{1} \in S(x /(p), y)} 1 \\
& =\frac{1}{\Omega_{z}\left(m_{1}\right)=k} \\
(k+1) & \sum_{p \leq z} N_{k}(x / p, y, z)
\end{aligned}
$$

By the assumption for $\Omega_{z}(n)=k$ and (18), we get

$$
\begin{aligned}
N_{k+1}(x, y, z) & \leq \frac{A\left(\log _{2} z+B\right)^{k}}{\log z(k+1) !} \sum_{p \leq z} \Psi(x / p, y) \\
& =\frac{A \Psi(x, y)}{\log z} \frac{\left(\log _{2} z+B\right)^{k}}{(k+1) !} \sum_{p \leq z} \frac{1}{p^{\alpha}}\left\{1+O\left(\frac{1}{u}\right)\right\} .
\end{aligned}
$$

By applying the estimate in (66), we arrive at the following bound for $N_{k+1}(x, y, z)$

$$
N_{k+1}(x, y, z) \leq \frac{A \Psi(x, y)}{\log z} \frac{\left(\log _{2} z+B\right)^{k+1}}{(k+1) !},
$$

so we derived our desired result.

Proof of Theorem 1.3. For a small $\epsilon>0$, we set $u<\left(\frac{\lambda}{\log 2}-\epsilon\right) \log _{2} y$, where $\lambda$ is a fixed real number in the open interval $(1-2 \log 2,1-\log 2)$.

We now set $z$ satisfying

$$
\log \log z=\frac{\log 2}{\lambda} u
$$


so the given ranges of $u$ and $z$ satisfy the conditions of Lemma 4.1.

By the definition of $A(x, y)$, we have the following evident bound of $A(x, y)$

$$
A(x, y) \leq \sum_{k} \min \left\{\sum_{\substack{n \in S(x, y) \\ \Omega_{z}(n)=k}} 1, \sum_{\substack{j=1 \\ \sum_{a \in S(\sqrt{x}, y)}}} 1 \sum_{\substack{b \in S(\sqrt{x}, y) \\ \Omega_{z}(a)=j}} 1\right\} .
$$

We set

$$
L=\left\lfloor H \log _{2} z\right\rfloor,
$$

where

$$
H:=\frac{1-\lambda}{\log 2} .
$$

We have $1-2 \log 2<\lambda<1-\log 2$. Thus, $1<H<2$.

By using (69), we write the following bound for $A(x, y)$

$$
\begin{aligned}
A(x, y) & \leq \#\left\{n \in S(x, y): \Omega_{z}(n)>L\right\}+\#\left\{a b: a, b \in S(\sqrt{x}, y), \Omega_{z}(a)+\Omega_{z}(b) \leq L\right\} \\
& =\sum_{k>L} N_{k}(x, y, z)+\sum_{k \leq L} \sum_{j=0}^{k} N_{j}(\sqrt{x}, y, z) N_{k-j}(\sqrt{x}, y, z) .
\end{aligned}
$$

By applying Lemma 4.1, we have

$$
\begin{aligned}
A(x, y) & \ll \sum_{k>L} \frac{\Psi(x, y)}{\log z} \frac{\left(\log _{2} z+c\right)^{k}}{k !}+\sum_{k \leq L} \sum_{j=0}^{k} \frac{\Psi^{2}(\sqrt{x}, y)}{\log ^{2} z} \frac{\left(\log _{2} z+c\right)^{j}}{j !} \frac{\left(\log _{2} z+c\right)^{k-j}}{(k-j) !} \\
& =\sum_{k>L} \frac{\Psi(x, y)}{\log z} \frac{\left(\log _{2} z+c\right)^{k}}{k !}+\sum_{k \leq L} \frac{\Psi^{2}(\sqrt{x}, y)}{\log ^{2} z} \sum_{j=0}^{k} \frac{1}{k !}\left(\begin{array}{c}
k \\
j
\end{array}\right)\left(\log _{2} z+c\right)^{k} \\
& =\sum_{k>L} \frac{\Psi(x, y)}{\log z} \frac{\left(\log _{2} z+c\right)^{k}}{k !}+\sum_{k \leq L} \frac{\Psi^{2}(\sqrt{x}, y)}{\log ^{2} z} \frac{\left(2 \log _{2} z+c\right)^{k}}{k !} .
\end{aligned}
$$

By applying the simple form of $\Psi(x, y)$ in Corollary 2.2, and using the assumption (68), we get

$$
\frac{\Psi^{2}(\sqrt{x}, y)}{\Psi(x, y)} \asymp(\log z)^{\lambda} \quad \text { as } \quad u, y \rightarrow \infty .
$$

Thus,

$$
A(x, y) \ll \frac{\Psi(x, y)}{\log z} \sum_{k>L} \frac{\left(\log _{2} z+c\right)^{k}}{k !}+\frac{(\log z)^{\lambda} \Psi(x, y)}{\log ^{2} z} \sum_{k \leq L} \frac{\left(2 \log _{2} z+c\right)^{k}}{k !} .
$$

The maximum values of functions in the above summands (with respect to $k$ ) are attained at $k=\left\lfloor\log _{2} z\right\rfloor$ and $k=\left\lfloor 2 \log _{2} z\right\rfloor$ respectively. We have $\log \log z<L<2 \log \log z$, so the function in the first summation in (73) in decreasing for $k>L$, and by using Stirling's formula $k ! \sim k^{k+\frac{1}{2}} e^{-k}$, we have 


$$
\begin{aligned}
\sum_{k>L} \frac{\left(\log _{2} z\right)^{k}}{k !} & =\sum_{H \log _{2} z<k \leq e \log _{2} z} \frac{\left(\log _{2} z\right)^{k}}{k !}+\sum_{e \log _{2} z<k \leq 2 e \log _{2} z} \frac{\left(\log _{2} z\right)^{k}}{k !}+\sum_{k>2 e \log _{2} z} \frac{\left(\log _{2} z\right)^{k}}{k !} \\
& \ll\left(\log _{2} z\right)\left(\left(\frac{e}{H}\right)^{H \log _{2} z}+1\right) \\
& \ll \frac{1}{(\log z)^{H \log H-H}} .
\end{aligned}
$$

The function in the second summation in (73) is increasing for $k \leq L$, and we have

$$
\sum_{k \leq L} \frac{\left(2 \log _{2} z+c\right)^{k}}{k !} \ll\left(\log _{2} z\right)\left(\frac{2 e}{H}\right)^{H \log _{2} z}=\frac{1}{(\log z)^{H \log H-H-H \log 2}}
$$

Substituting the upper bounds obtained in (74) and (75) in (73), and using the definition of $H$, gives

where

$$
A(x, y) \ll \frac{\Psi(x, y)}{(\log z)^{G(H)}},
$$

$$
G(H):=1+H \log H-H .
$$

The function $G(H)$ is an increasing function in the interval $(1,2)$ with a zero at $H=1$. Thus, for any arbitrary $1-2 \log 2<\lambda<1-\log 2$, we have

$$
A(x, y)=o(\Psi(x, y)) \quad \text { as } \quad x, y \rightarrow \infty,
$$

so we obtained our desired result.

\section{REFERENCES}

[1] Krishnaswami Alladi. An Erdős-Kac theorem for integers without large prime factors. Acta Arith., 49(1):81$105,1987$.

[2] William D. Banks and David J. Covert. Sums and products with smooth numbers. J. Number Theory, 131(6):985-993, 2011.

[3] E. R. Canfield, Paul Erd" os, and Carl Pomerance. On a problem of Oppenheim concerning "factorisatio numerorum". J. Number Theory, 17(1):1-28, 1983.

[4] Régis de la Bretèche and Gérald Tenenbaum. Propriétés statistiques des entiers friables. Ramanujan J., 9(1-2):139-202, 2005.

[5] P. Erdős. An asymptotic in the theory of numbers.(russian). Vestnik Leningrad. Univ., 15(13):41-49, 1960.

[6] Paul Erdös. Some remarks on number theory. Riveon Lematematika, 9:45-48, 1955.

[7] Kevin Ford. Integers with a divisor in $(y, 2 y]$. In Anatomy of integers, volume 46 of CRM Proc. Lecture Notes, pages 65-80. Amer. Math. Soc., Providence, RI, 2008.

[8] John B. Friedlander. Integers free from large and small primes. Proc. London Math. Soc. (3), 33(3):565-576, 1976.

[9] Andrew Granville. Smooth numbers: computational number theory and beyond. In Algorithmic number theory: lattices, number fields, curves and cryptography, volume 44 of Math. Sci. Res. Inst. Publ., pages 267-323. Cambridge Univ. Press, Cambridge, 2008.

[10] Adolf Hildebrand. On the number of positive integers $\leq x$ and free of prime factors $>y$. J. Number Theory, 22(3):289-307, 1986.

[11] Adolf Hildebrand. On the number of prime factors of integers without large prime divisors. J. Number Theory, 25(1):81-106, 1987. 
[12] Adolf Hildebrand and Gérald Tenenbaum. On integers free of large prime factors. Trans. Amer. Math. Soc., 296(1):265-290, 1986.

[13] Éric Saias. Entiers sans grand ni petit facteur premier. II. Acta Arith., 63(4):287-312, 1993.

[14] Éric Saias. Entiers sans grand ni petit facteur premier. III. Acta Arith., 71(4):351-379, 1995.

[15] Gérald Tenenbaum. Introduction to analytic and probabilistic number theory. Cambridge University Press, 1995.

Départment de Mathématiques et Statistique,Université de Montréal, CP 6128, succ. Centreville, Montréal, QC, Canada H3C 3J7.

E-mail address: marzieh.mehdizadeh@gmail.com 